\title{
Recent developments in neoantigen-based cancer vaccines
}

\author{
Kenneth Hodge, ${ }^{1, \#}$ Suangson Supabphol, ${ }^{1, \#}$ Pramod Kumar, ${ }^{2, \#}$ Witthaya Poomipak, ${ }^{3}$ Trairak Pisitkun ${ }^{1}$
}

\begin{abstract}
Cynics point out that a cure for cancer has been "around the corner" for the last 50 years. Nevertheless, the recent convergence of deep DNA, RNA, and proteomic technologies with enhanced understanding of the nuances of the adaptive immune system has generated great optimism amongst researchers. The extraordinary heterogeneity of various cancers, once thought to be a major therapeutic hurdle, may now be bypassed via "personalized" vaccine treatments. Specifically, these treatments involve the identification of MHC-bound peptides that are unique to a patient's cancer (neoantigens), followed by immunization with peptides, RNA, or DNA that encodes these neoantigens via various delivery systems, thus amplifying the immune system's response to the particular cancer. Such approaches have shown dramatic results in animal studies. Not surprisingly, then, the field of neoantigen-based immunotherapy has advanced at a spectacular rate, necessitating that interested individuals stay apprised of recent developments. Following an introduction to the subject, we thus focus on aspects that are particularly fast-moving; the cellular sources of neoantigens, which are surprisingly diverse, the tools that are used for their identification, and the status of the numerous clinical trials that are now being conducted.
\end{abstract}

Key words: cancer, vaccine, immunotherapy, bioinformatics, clinical trials

\footnotetext{
From:

1 The Center of Excellence in Systems Biology, Faculty of Medicine, Chulalongkorn University, Bangkok, Thailand

2 The Program in Bioinformatics and Computational Biology, Graduate School, Chulalongkorn University, Bangkok, Thailand

3 The Department of Research affairs, Faculty of Medicine, Chulalongkorn University, Bangkok, Thailand

\# These authors contributed equally to this work
}

Corresponding author:

Trairak Pisitkun

The Center of Excellence in Systems Biology, Faculty of Medicine, Chulalongkorn University, 1873 Rama 4 Road, Pathumwan, Bangkok 10330, Thailand

E-mail: trairak.p@chula.ac.th

\section{List of abbreviations:}

aeTSA aberrantly expressed Tumor Specific Antigen

CTLA-4 Cytotoxic T-Lymphocyte-associated Protein 4

DC Dendritic Cell

dsRNA double-stranded RNA

GI Gastrointestinal

$\mathrm{H} \& \mathrm{~N} \quad$ Head and Neck

hERV human Endogenous Retrovirus

HLA Human Leukocyte Antigen

HLA-LOH HLA Loss of Heterozygosity

IFN Interferon

MHC Major Histocompatibility Complex

MS Mass Spectrometry

ncRNA Noncoding RNA

NK Natural Killer (cell)

NMD Nonsense-Mediated Decay

PD-1 Programmed Cell Death Protein 1

PD-L1 Programmed Cell Death Protein Ligand 1

RI

\begin{tabular}{ll}
\multicolumn{2}{l}{ List of abbreviations (Continued): } \\
SCLC & Small Cell Lung Carcinoma \\
SCM & Splice Site Creating Mutations \\
SNV & Single Nucleotide Variant \\
SSC & Splice Site Creation \\
TAA & Tumor-Associated Antigen \\
TLR & Toll-like Receptor \\
TMB & Tumor Mutation Burden \\
TNBC & Triple Negative Breast Cancer \\
TSA & Tumor specific Antigen \\
VEGF & Vascular Endothelial Growth Factor \\
WES & Whole Exome Sequencing
\end{tabular}

\section{Introduction}

The need for therapies that target cancers with high specificity has long been recognized. Early efforts in targeted therapy focused largely on tumor-associated antigens (TAAs). These proteins clearly served as markers for the presence of various cancers, but non-negligible expression in healthy tissue could not be ruled out. The presence of TAAs in healthy tissue likely explains several disastrous failures in the field. ${ }^{1-3}$ Even in the absence of dramatic side effects, the disappointing efficacy of some TAA-targeting therapies may now be explained by a stronger understanding of thymic selection, the process by which expression of a large portion of human proteins within the thymus enables self-tolerance to such proteins. ${ }^{4,5}$ The ideal target for therapy, then, would be one that arises via cancer's well-known predilection for high mutation rates or, at the very least, a target whose cancer-unique signature can be confidently inferred. Such targets are known as neoantigens. In addition to the obvious logic of focusing on neoantigen-based therapies, 
a number of studies revealed that the specific $\mathrm{T}$ cells responsible for some clinical responses recognized neoantigens. ${ }^{6}$ In parallel, other studies noted that the efficiency of checkpoint inhibitors, drugs that counter a number of immune-evasion tactics seen in cancers, often correlated with cancer "mutation load"; once the immune blockade has been lifted, more neoantigens mean more candidates for $\mathrm{T}$ cell recognition., Obviously, identification of a patient's very particular "mutanome" has only been feasible in the last decade or so, with the advent of cheap, accurate, and rapid exome sequencing.

Neoantigen-therapy is notable for the extent to which a patient's immune system is coaxed to perform the dirty work of cancer-killing. The essential steps of this process, applicable to any epitopes that are recognized as "foreign", are illustrated in Figure 1. We first note the heterogeneity of cancer. This heterogeneity may refer to DNA mutations or RNA and protein expression differences between cancer types (e.g. breast cancer vs. leukemia), or it may refer to intra-tumor differences. ${ }^{9}$ In any case, a near-unique repertoire of neoantigens is associated with a patient's cancerous tissue. Such antigens may be released into the blood upon cell death, and/or displayed on the cancer cell surface in accordance with the peptide-binding properties of the patient's set of HLA molecules. In the former case, antigen processing and display by dendritic cells serves to activate CD8 and CD4 T cells that survived thymic selection. $\mathrm{T}$ cell trafficking, a process often negatively regulated by cancers, ${ }^{10}$ then directs the $\mathrm{T}$ cells to the cancerous tissue, where interactions with HLA-displayed neoantigens initiate the process of cancer cell killing. ${ }^{11}$ Cancer-related antigens are then released into the blood, completing the "cancer immune cycle". Such positive feedback suggests that a simple "jump start", provided with or without the assistance of various therapies, may be sufficient for an effective cancer response. Inhibition of the cycle is, of course, a tactic employed by cancers. However, a healthy immune system also employs checkpoints to minimize the possibility of autoimmune responses.

\section{The Nuts and Bolts of Neoantigen Identification}

An early, two-step schema for the identification of neoantigens is seen in work from the lab of Catherine $\mathrm{Wu} .{ }^{12}$ The only essential "omics-level" wet-lab protocol was the whole-exome sequencing of both cancerous and germ-line tissue. Sequence

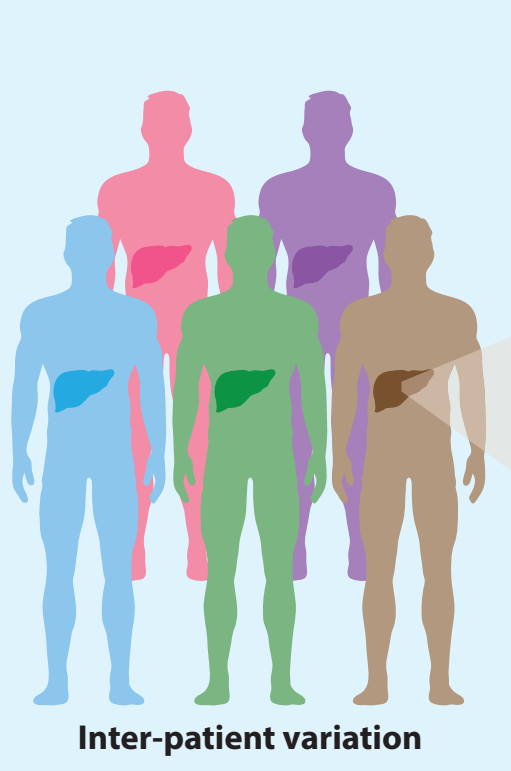

The cancer immune cycle

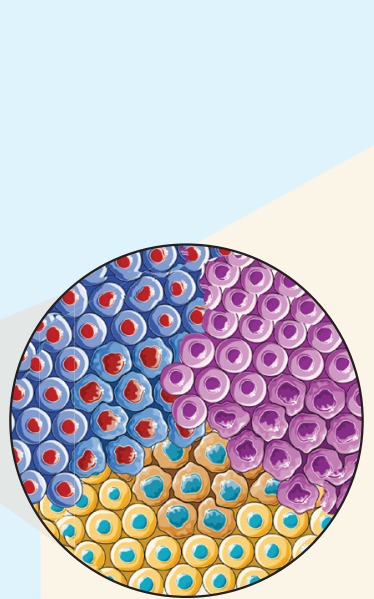

1. Mutational heterogeneity

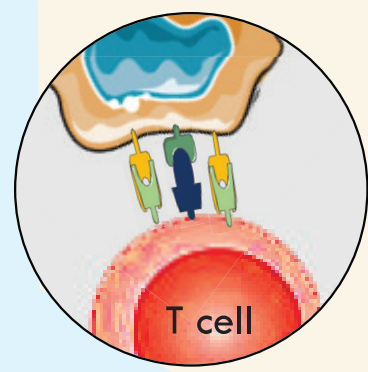

6. Cancer cell killing

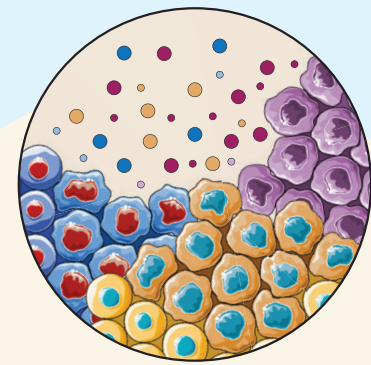

2. Cancer neoantigen release

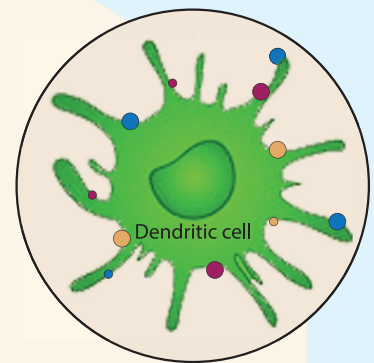

3. Cancer neoantigen presentation

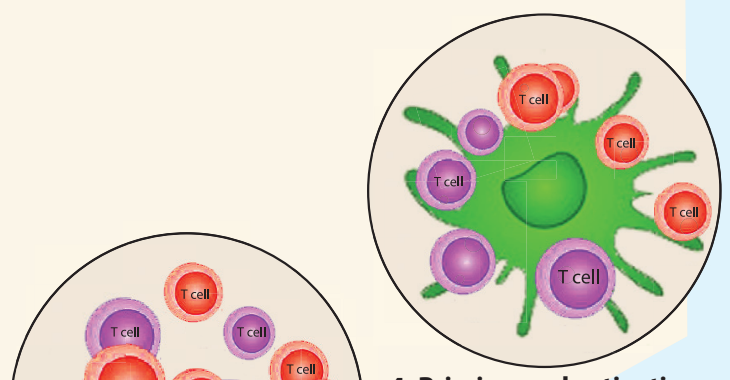

4. Priming and activation

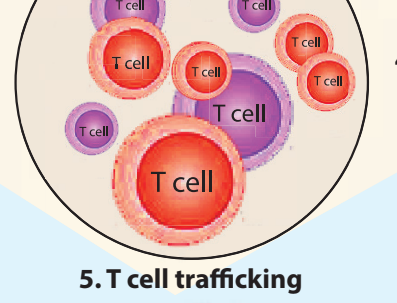

Figure 1. The cancer immune cycle. Throughout the image, different colors represent variation at different levels of the cancer immune response. Patients display great variation in their cancer mutanomes, even between shared cancer types (liver cancer is depicted here). Within a tumor, different colors show mutanome variability, with different tumor clones releasing neoantigens. At the level of the immune response, vast variation (for simplicity, only two different $\mathrm{T}$ cell clones are shown) in the T cell repertoire allows for recognition of neoantigens, followed by cancer cell targeting and killing. 
comparison allowed identification of cancer-specific non-synonymous mutations via the variant-calling tool, "Mutect."13 Next, neoantigen candidates were computationally screened for HLA-binding affinity. RNA-sequencing was performed only to validate the expression of particular neoantigen candidates. ELISpot assays were performed, with IFN- $\gamma$ release signaling that a particular neoantigen elicited a T cell response.

Even with the most minimal of protocols, confounding factors arise. In particular, we note the necessity of calling a patient's specific HLA repertoire amongst the thousands of variants in the human population in order to predict the efficacy of particular neoantigens. Also, given cancer heterogeneity, the likelihood of healthy tissue contaminating cancer samples, and possible inaccuracies in sequencing data, defining and refining the cutoffs at which a mutant may be confidently identified is not a trivial exercise. Currently, a number of tools are available to simplify the process of HLA-typing (see the following reference for a comparison of these tools) ${ }^{14}$ and to assess tumor heterogeneity and contamination. ${ }^{15}$

Deep RNA sequencing is now routine in most neoantigenidentification pipelines. Given the potential volume of neoantigen candidates identified in the process of variant calling, it is convenient to validate RNA expression prior to HLA-binding assessment. Also, as will be seen below, researchers may wish to examine the possibility of neoantigens being generated from genomic regions not covered in standard exome sequencing.

Mass spectrometry (MS) is also often utilized in neoantigen identification. Given that neoantigens are peptides, not nucleotide sequences, MS usage is of obvious logic. One might even ask why exome- and RNA-seq are needed in a universe where peptides in cancerous tissue can be compared with peptides in healthy tissue. A number of issues impair the utility of MS, however. Most importantly, MS currently lacks the sensitivity to detect low-abundance peptides. Also, the copious quantities of HLA-specific antibodies required for peptide isolation are problematic. Finally, the apparent absence of a peptide in healthy tissue does not guarantee a system-wide absence. One technology finding increased prominence in neoantigen identification protocols is that of ribosome profiling, which enriches for RNAs that are under translation, revealing the likely translation frame. In some sense, then, "RiboSeq" bridges the gap between RNA-seq and MS.

Figure 2 illustrates a current, basic path toward neoantigen vaccine application. In addition to the bioinformatics-intensive steps discussed above, it is seen that decisions regarding neoantigen delivery remain. Also, appropriate adjuvants that can generate satisfying anti-tumor $\mathrm{T}$ cell responses must be chosen. Current trends in both areas are noted below in the clinical trials sections.

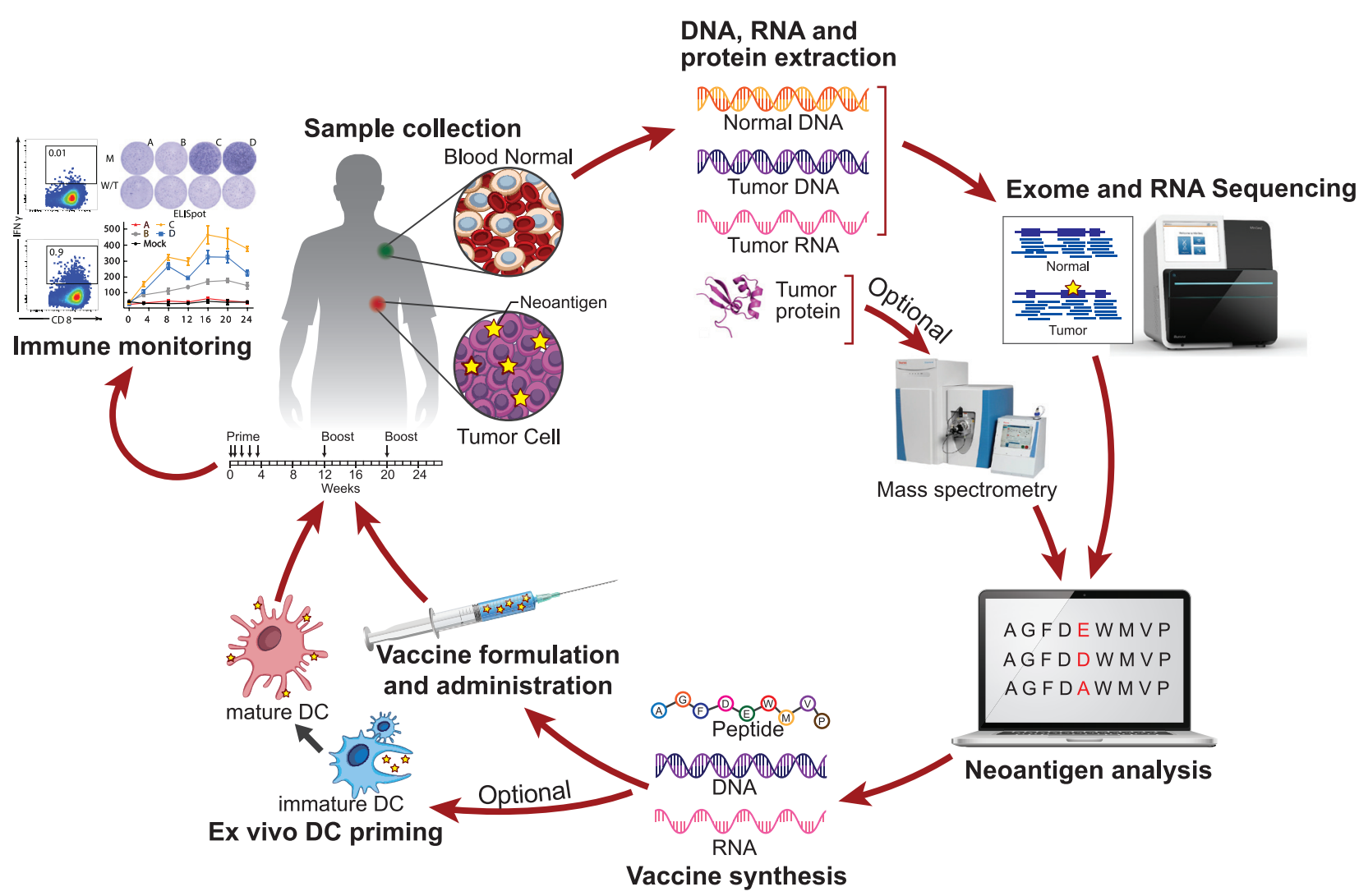

Figure 2. A simple, standard workflow from patient sampling through treatment. Patient cancer DNA is compared with DNA from healthy tissue. RNA sequencing assures that neoantigens of interest are expressed. Mass spectrometry may confirm that neoantigens are bound to HLA. Vaccines may be formulated as peptides, or as the RNA or DNA that encodes the peptide. As with vaccines against infectious agents, adjuvants are typically included in the formulation. Peptides may also be incubated with isolated dendritic cells in order to activate these cells ex vivo before vaccination with these dendritic cells. Note that a checkpoint inhibitor drug is often administered to patients to help alleviate immunosuppression in the tumor microenvironment. 


\section{Sources of Neoantigens}

Protocols for neoantigen identification have, over their short history, focused on cancer-specific mutations within protein-coding regions of DNA, namely non-synonymous single -nucleotide variants (SNVs) and indels. ${ }^{16-18}$ This approach is both logical and convenient, as therapeutic effects depend on cancer-specific display of peptides, and exome-sequencing is now routinely performed without great expense. Nevertheless, numerous researchers have focused on the identification of non-canonical sources of neoantigens. Such a search is necessitated by several factors. Most importantly, following computational winnowing of poorly-displayed and poorly-immunogenic canonical neoantigens, some patients are left without a single vaccine candidate. In one summary of 13 studies, 53 of 1,874 tested SNV neoantigens were shown to elicit a $\mathrm{T}$ cell response; on average, fewer than 2 neoantigens per patient were immunogenic. ${ }^{19}$ Thus, additional sources of neoantigens may be necessary in a large number of cases. Also, it is expected that some classes of neoantigens should provoke a stronger immune response than others. For example, a standard SNV-derived neoantigen is characterized by a single amino acid alteration which, given the nature of HLA display and the genetic code, may not differ dramatically from its wildtype relative. A more exotic class of neoantigens, on the other hand, may lack similarity to any peptide found within the healthy proteome, thus possibly provoking a heightened immune response. Finally, though the vast majority of canonical neoantigens are "personal", i.e. found only on a patient-bypatient basis, other classes may be shared amongst patients, raising the possibility of "off-the-shelf" treatments.

Potential sources of neoantigens are listed in Figure 3. Following canonical neoantigen sources, we list fusions, the cancer-specific linkage of regions normally found on separate chromosomes. Such events should frequently give rise to

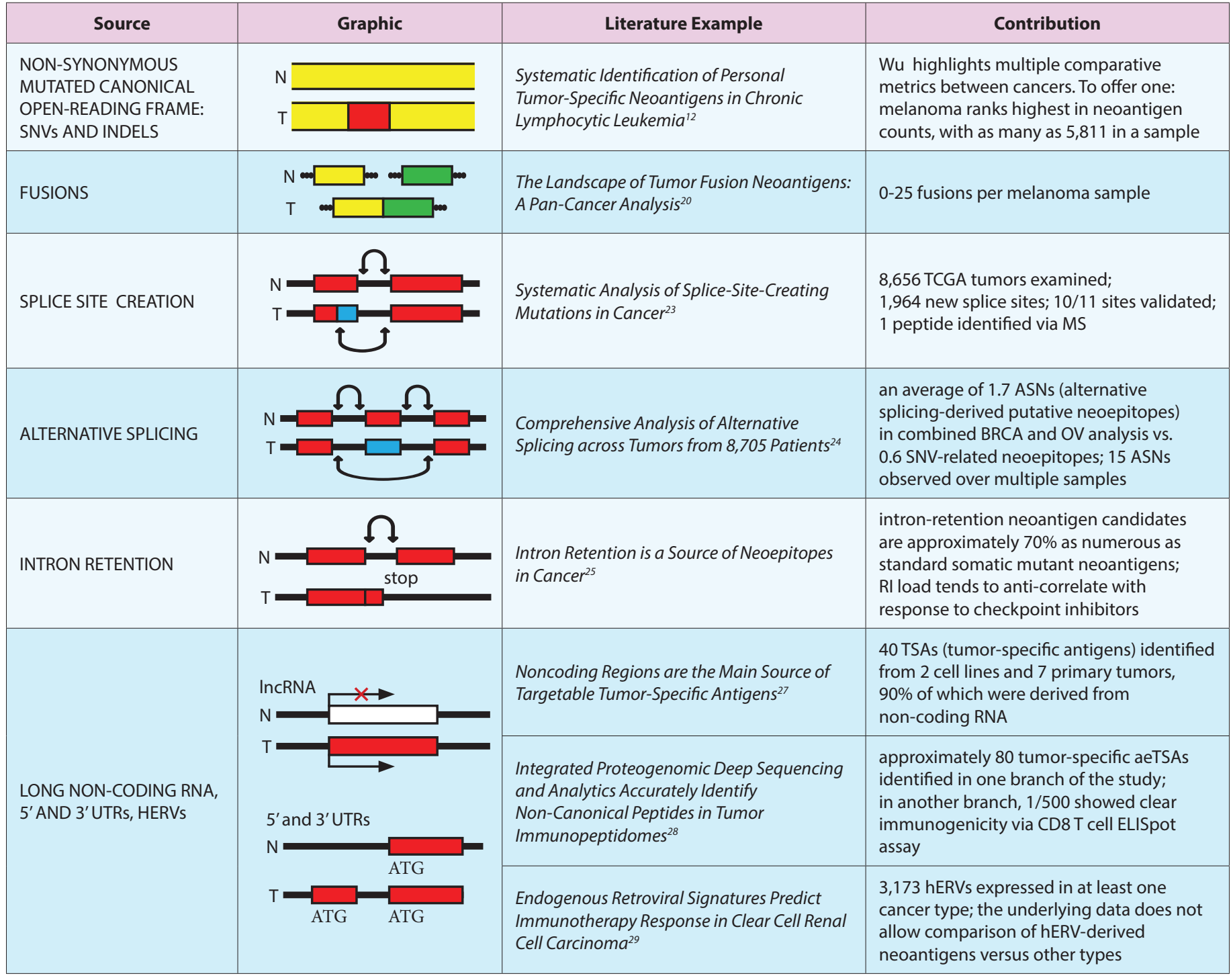

Figure 3. Potential sources of neoantigens, prime examples of literature describing their discovery, and some data illustrating the possible contribution of these neo-antigens at the per-patient level. Full titles are given in the "literature example" column to give readers a sense of the subject. For graphics, dotted lines indicate a continuing sequence. PTM; post-translational modification; $\mathrm{N}$; normal tissue, T; tumor tissue, P; phosphorylation. A typical RNA-editing event is depicted, with adenosine altered to inosine. In the final two rows, readers should be aware that neoantigens are generated at the post-translational level, i.e. representations of peptides are shown. 


\begin{tabular}{|c|c|c|c|}
\hline Source & Graphic & Literature Example & Contribution \\
\hline $\begin{array}{l}\text { RNA EDITING AND } \\
\text { MODIFICATION }\end{array}$ & $\begin{array}{l}\mathrm{N} \\
\mathrm{T} \\
\mathrm{I}\end{array}$ & $\begin{array}{l}\text { A-to-I RNA Editing Contributes to Proteomic } \\
\text { Diversity in Cancer }{ }^{31}\end{array}$ & no evidence of cancer-specific expression \\
\hline CANCER-SPECIFIC PTMS & $\begin{array}{l}\mathrm{N} \square \\
\mathrm{T} \square \mathrm{T} \\
\square\end{array}$ & $\begin{array}{l}\text { Mass Spectrometry Based } \\
\text { Immunopeptidomics Leads to Robust } \\
\text { Predictions of Phosphorylated HLA Class I } \\
\text { Ligands }^{33}\end{array}$ & $\begin{array}{l}2,190 \text { phosphorylated HLA-I ligands } \\
\text { identified without phospho-enrichment } \\
\text { from } 61 \text { samples }\end{array}$ \\
\hline \multirow{3}{*}{ PROTEASOME SPLICING } & \multirow{3}{*}{ (2) } & $\begin{array}{l}\text { Mapping the MHC Class I Spliced } \\
\text { Immunopeptidome of Cancer Cells } s^{36}\end{array}$ & \multirow{3}{*}{ no evidence of cancer-specific expression } \\
\hline & & $\begin{array}{l}\text { Estimating the Contribution of Proteasomal } \\
\text { Spliced Peptides to the HLA-I Ligandome }{ }^{37}\end{array}$ & \\
\hline & & $\begin{array}{l}\text { Global Identification of Post-Translationally } \\
\text { spliced Peptides with Neo-Fusion }\end{array}$ & \\
\hline
\end{tabular}

Figure 3. (Continued)

neoantigens at junctions, and potentially downstream of junctions in cases where a frame-shift results. In one study, ${ }^{20}$ these neoantigens showed relatively high immunogenicity, though shared neoantigens and driver neoantigens tended to show low immunogenic potential. As many as 55 fusions were identified in a single cancer sample. At least two studies have demonstrated immunogenicity for specific fusion-derived neoantigens. ${ }^{21,22}$ Vaccine candidates based on fusions pass the "common sense" test, but are limited by a relative paucity of fusion events versus SNVs and indels in cancer.

The next two sources of neoantigens that we examine both relate to splicing. In the case of splice site creation (SSC), cancer-specific mutations create new unique splice sites and thus neoantigens. In the case of cancer-related alternative splicing, mutations near existing splice sites or within splicing proteins increase the extent to which exons are retained or skipped. The two cases differ in the extent to which one can confidently assume that a potential neoantigen is truly unique to a cancer. In the case of SSC, entirely new exons are found within the final spliced RNA. In the case of cancer-related alternative splicing, however, it is essentially impossible to conclude that a particular alternate splicing arrangement is unique to cancer tissue. Splice-site-creating mutations (SCMs) were investigated by Jayasinghe et al. ${ }^{23}$ Bearing in mind that a single SCM can be responsible for multiple neoantigens, 23\% of 8,656 tumor samples expressed SCMs. A number of SCMs were recurrent. One particular recurrent SCM, found within the GATA3 gene, was singled out and validated via MS-based discovery. Regarding the potential of alternatively-spliced TSAs, there are reasons for optimism despite the aforementioned caveat. In the premier work on the subject, ${ }^{24}$ Kahles et al scoured TCGA data and found nearly 3 predicted HLA-binding "neojunction" peptides for every such SNV-derived peptide. Furthermore, a surprisingly high number of neojunctions were recurrent; of nearly 9,000 samples, $7 \%$ of neojunctions were seen in more than 100 samples.

Though associated with splicing, we list "intron retention" (RI) as a separate source of neoantigens. Here, failure of splicing machinery or splice-site mutations causes introns to be included in mature RNA, generating neoantigens. In the case of mutation-associated RI, the confidence that the candidate neoantigen is unique to the cancer would be heightened. A study focusing on intron retention in melanoma tissue was able to increase neoantigen load by $70 \%$ via the inclusion of $\mathrm{RI}^{25}$ versus canonical somatic mutation neoantigens. Naively, one might expect that nonsense-mediated decay (NMD) would minimize the presence of RI neoantigens. In fact, one broad screen of TCGA data suggests a general trend toward enhancement, as opposed to dysregulation, of NMD in cancer. ${ }^{26}$ Nevertheless, a number of these candidates could indeed be identified via MS, though perhaps oddly, a slight anti-correlation was noted between RI load and checkpoint inhibitor benefit.

In examining the potential for neoantigen expression via non-coding RNAs (ncRNA), Laumont et al tackled the differential expression problem by using thymic RNA as the control tissue. ${ }^{27}$ The premise here is that the thymus expresses a large percentage of known genes given its role in inducing tolerance to HLA-displayed peptides. If identical reads were seen in both cancer and thymic tissue, the reads were excluded. Over a limited number of human cancer samples (four B-ALLs and three lung cancers), 20 of 22 MS-validated tumor specific antigens (TSAs) were not mutation-associated. Rather, these were non-mutated peptides derived from non-coding regions that were aberrantly expressed in cancer (aeTSAs). Similar percentages were derived from the mouse branch of the study, where immunization with TSA-pulsed dendritic cells showed dramatic increases in mouse survival in several cases. It is thus clear that therapy using aeTSAs may provoke life-saving immune responses. Additionally, given that aeTSAs are not the result of mutation, they are expected to be recurrent over multiple patients; recurrent aeTSAs were indeed identified in Laumont's work. Bassani-Sternberg et al examined the possibility of non-coding RNA as a source of aeTSAs using a different approach. ${ }^{28}$ Here, ribosomal profiling was used to assay RNAs with which ribosomes interact. Such a method allows the prediction of reading frames, dispensing with the need for in-silico three-frame translation of all RNAs found to be dominant in tumor tissue. It also allows 5' and 3' UTRs to be examined for non-canonical translation. Stringent MS analysis of tumor samples versus controls followed, culminating in the identification of about 80 aeTSAs that could not be identified in healthy tissue. A comparison of canonical versus non-canonical TSA yield was only made for several samples, yielding an approximate 1:1 ratio of both. However, of greater than 
500 aeTSAs screened, only one displayed strong immunogenicity, with authors explaining that such results may reflect difficulties inherent in current screening protocols. Again, the possibility of shared antigens was emphasized. A final recent work examines a subset of ncRNA, that of endogenous retroviral elements (hERVs), again using ribosomal profiling to home in on regions that may be translated. ${ }^{29}$ To no surprise, peptides generated from hERVs indeed showed evidence of translation, with tetramer assays showing that peptides derived from a specific hERV, hERV-4700, were recognized by a large subset of infiltrating T-cells. A new tool for the identification of hERVs from RNA-seq data, hervQuant, is provided in the study.

We conclude our examination of TSA sources with three rather exotic sources of potential antigens. In all three cases, cancer immunogenicity would depend on broad biological mechanisms being specifically altered in cancer tissue. For example, RNA-editing is a process by which the sequence of RNA may be altered after transcription. This editing is performed by a limited array of enzymes, most prominent of which is ADAR. ${ }^{30}$ Though RNA-editing alterations have indeed been recognized in cancer, ${ }^{31}$ a clear "cancer only" peptide candidate has yet to be associated with RNA-editing. Similarly, it is possible that a peptide sequence may be displayed in both normal and cancer tissue, but only the cancer peptide receives, or lacks, a particular post-translational modification. It is clear that modified peptides may be displayed by HLA molecules, ${ }^{32,33}$ but few reports suggesting cancer-specific immunogenicity exist. In one case, glycosylated peptides associated with leukemia could stimulate ELISpot IFN- $\gamma$ signals in PBMCs from healthy donors. ${ }^{34}$ Finally, proteasomal splicing is a process by which two independent peptides may actually be ligated after proteasomal cleavage of their original proteins. It is clear that such peptides may be displayed by HLA. ${ }^{35-38}$ Once again, however, the evidence for neoantigens derived from the splicing of peptides is lacking.

We've refrained from listing one final intriguing, potential class of neoantigens in our table, as their utility in vaccines remains questionable: fragmented proteins that are unique to apoptotic cancer cells. ${ }^{39}$ These caspase-cleavage products have been described as immunogenic, but it remains to be seen if vaccination against a class of peptides generated from cells that are essentially dissolving could be effective. Certainly, "bystander" effects, whereby released antigens are picked up for display on neighboring cells, have been described. ${ }^{40}$

\section{Pre-Clinical Studies}

Published pre-clinical studies have demonstrated significant $\mathrm{T}$ cell responses after neoantigen-based vaccinations in mice. Yadav et al. injected C57BL/6 mice with MC-38 colon cancer cells and examined the response to neoantigen vaccination in both prophylactic and therapeutic settings. ${ }^{41} \mathrm{Neo}-$ epitopes were derived via MC38 exome sequencing, MHC-I binding and solvent-exposure analysis of candidate neoantigens, followed by mass spectrometric verification. In the prophylactic model, tumor volumes significantly decreased. In two cases where tumor growth was not inhibited, dextramer staining revealed low levels of neoantigen-specific CD8 T cells, suggesting that CD8 $\mathrm{T}$ cells were responsible for protection against MC-38 tumor growth in these mice. Significant tumor growth inhibition was also observed in the therapeutic setting. It was also shown that neoantigen-reactive CD8 T cells and IFN- $\gamma$ expression increased within tumors after vaccination. Castle et al. also demonstrated tumor regression in C57BL/6 mice, this time inducing tumors with the B16F10 melanoma cell line. ${ }^{42}$ Survival was also examined, with $40 \%$ of the mice

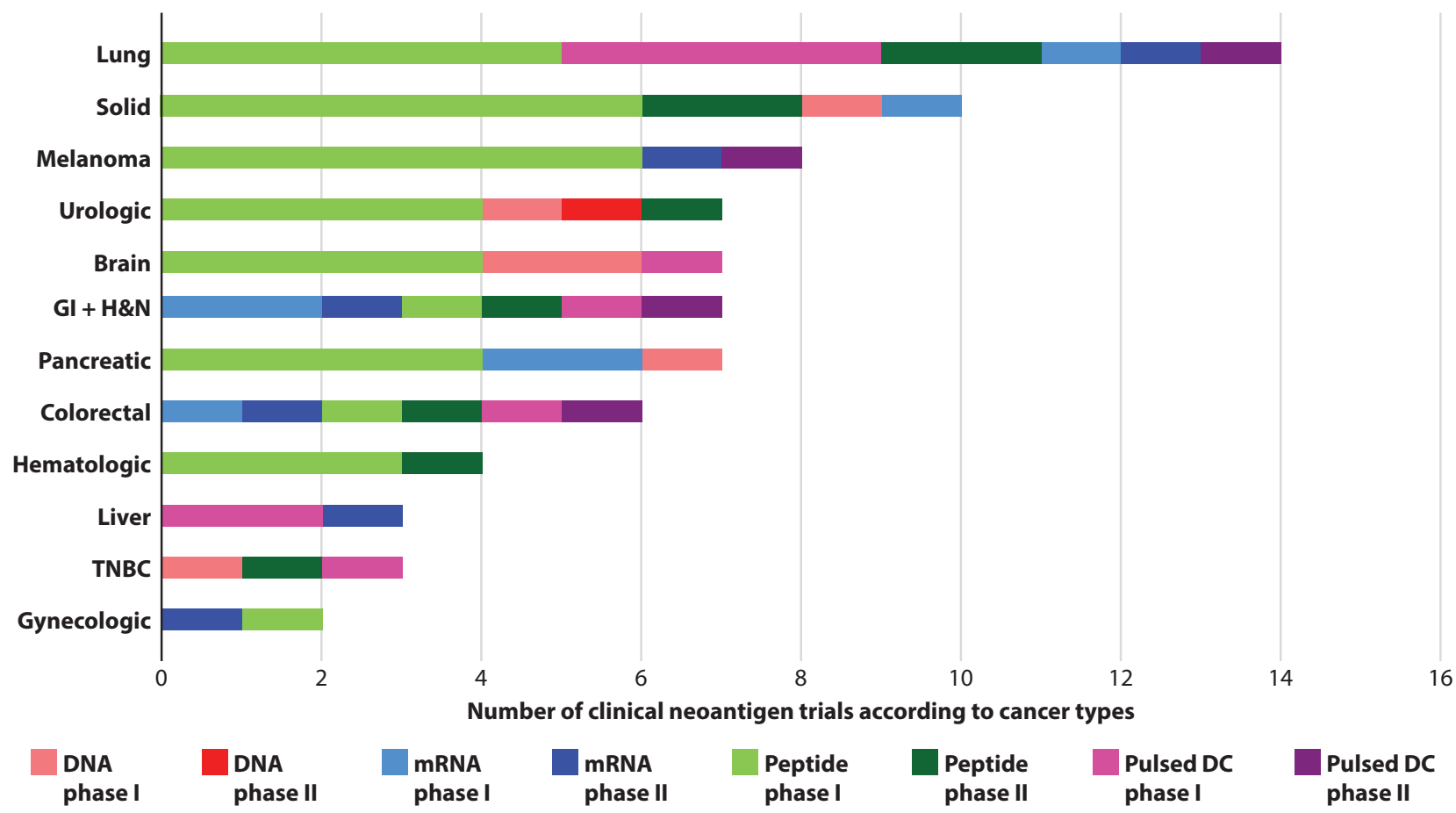

Figure 4. Clinical neoantigen trials according to cancer types. $\mathrm{X}$-axis indicates the number of current, ongoing trials. Coloring indicates both the vaccine formulation and the status of the trial. 
immunized with a particular neoantigen ("mut30") surviving, while all mice within the control-treatment group died within 44 days. Numerous other examples of neoantigen-related tumor regression and enhanced survival in mouse models are available..$^{27,43}$

\section{Clinical Studies}

Given clear positive outcomes in animal models, a large number of human clinical trials are currently ongoing. Registered "neoantigen" clinical trials in www.clinicaltrials.gov, updated April 2020, and sorted by cancer types, experimental phases, and delivery platforms, are illustrated in Figure 4. Of 59 trials, 46 trials are in phase I, while 13 trials have proceeded to phase II. Breaking the trials down by treatment modality, 35 utilize neoantigen peptides, 11 involve neoantigen priming of DCs, and 13 use DNA or mRNA-based vaccines. Studies focus on cancers with known high neoantigen loads, such as non-small cell lung cancer, melanoma, and bladder cancer.

Several recently completed clinical trials have shown promising results. Yong Fang et al. revealed that peptide-based vaccines utilizing a total of 10 to 20 different synthetic long peptides per patient, were safe and elicited effective $\mathrm{T}$ cell responses in multiple advanced cancers such as non-small cell lung cancer, pancreatic cancer, colorectal cancer, melanoma, ovarian cancer (trial: NCT03662815). In post-vaccination blood samples, $80 \%$ of patients had significant $\mathrm{T}$ cell responses as detected by ELISpot assays (unpublished abstract: https://c ancerres.aacrjournals.org/content/79/13_Supplement/CT006).
In another phase $1 \mathrm{~b}$ trial, peptide-based vaccines were introduced in combination with nivolumab to 10 metastatic melanoma patients (NCT02897765). Siwen Hu-Lieskovan et al. reported that $\mathrm{T}$ cell responses in post-vaccination blood samples were detectable in 12 out of 14 peptide treatments. The responses were durable up to 52 weeks after the first dose, with significant changes seen in TCR repertoire analysis (unpublished abstract: https://cancerres.aacrjournals.org/content/79/ 13_Supplement/4096) Song Gao et al. report similar results in the case of a Chinese patient with chemo-resistant small cell lung carcinoma (SCLC) with brain metastasis. The patient received autologous DC-based neoantigen cancer vaccines. After 10 months, all brain metastases regressed. ELISpot assays of post-vaccination blood samples revealed detectable CD4 and CD8 responses to 8 out of 12 neo-epitopes (unpublished abstract: https://cancerres.aacrjournals.org/content/79/13_Sup plement/942).

\section{Therapies combined with vaccines}

In the early stages of cancer, one modality of treatment, such as surgery, may possibly cure the disease. However, advanced cancers require multi-therapeutic agents such as chemotherapeutic, targeted, and immunotherapeutic drugs. ${ }^{44}$ In current clinical trials, personalized cancer vaccines are often combined with additional agents to maximize the potential to improve clinical outcomes. ${ }^{45}$ Supplementary agents used with personalized cancer vaccines in current clinical trials are seen in Table 1. Out of 59 trials, 28 combine neoantigen vaccines with either

Table 1. The agents currently used in combination with neoantigen therapy trials.

\begin{tabular}{|c|c|c|c|}
\hline Groups & Subgroups & Drugs & Number of trials \\
\hline \multirow{9}{*}{ Monoclonal antibody drugs } & \multirow{3}{*}{ Checkpoint inhibitors: Anti-PD-1 } & Nivolumab & 9 \\
\hline & & Pembrolizumab & 5 \\
\hline & & Toripalimab & 1 \\
\hline & \multirow{2}{*}{ Checkpoint inhibitors: Anti-PD-L1 } & Durvalumab & 3 \\
\hline & & Atezolizumab & 4 \\
\hline & \multirow{2}{*}{ Checkpoint inhibitors: Anti-CTLA-4 } & Ipilimumab & 8 \\
\hline & & Tremelimumab & 1 \\
\hline & Anti-CD20 & Rituximab & 1 \\
\hline & Anti-VEGF & Bevacizumab & 1 \\
\hline \multirow{9}{*}{ Chemotherapy drugs } & \multirow{3}{*}{ Anti-metabolites } & Gemcitabine & 2 \\
\hline & & 5-Fluorouracil & 1 \\
\hline & & Pemetrexed & 1 \\
\hline & \multirow{4}{*}{ Alkylating agents } & Carboplatin & 2 \\
\hline & & Oxaliplatin & 1 \\
\hline & & Cyclophosphamide & 2 \\
\hline & & Temozolomide & 1 \\
\hline & \multirow{2}{*}{ Plant alkaloids } & Nab-paclitaxel & 1 \\
\hline & & Irinothecan & 1 \\
\hline
\end{tabular}


immune checkpoint inhibitors, targeted therapies, or chemotherapeutic drugs, while 31 trials focus on neoantigen vaccines alone in order to evaluate safety and efficacy. In 12 trials, vaccines are coupled with more than one additional agent.

The most commonly used immune checkpoint inhibitors in clinical studies are anti-PD-1 agents (nivolumab and pembrolizumab), anti-PD-L1 (atezolizumab and durvalumab), and anti-CTLA-4 (ipilimumab). Immune checkpoint inhibitors are aimed at boosting $\mathrm{T}$ cell levels that have been exhausted in the tumor microenvironment. ${ }^{46}$ Combining vaccines with immune checkpoint inhibitors have shown promising results in both pre-clinical and clinical trials. ${ }^{47-49}$ A phase 2 clinical trial suggests that the combination of peptide-based vaccines with nivolumab significantly improves overall survival in patients with HPV-driven cancers. ${ }^{48}$ Not surprisingly, the response to immune checkpoint inhibitors is also determined by the mutational landscape; patients with high-tumor mutation burden (TMB) tend to have robust $\mathrm{T}$ cell reactivity and better clinical outcomes after receiving immune checkpoint inhibitors. ${ }^{50,51}$

Chemotherapeutic drugs are believed to promote tumor immunity in several ways. Immunogenic cancer cell death activates both the innate and adaptive immune responses via dsDNA and cancer antigen release, respectively. ${ }^{52}$ Moreover, chemotherapy is capable of modulating immunorecognition via various mechanisms, such as the upregulation of co-stimulatory molecules and downregulation of various immune checkpoint molecules. There is no significant preference for particular chemotherapeutic drugs used in the trials. Examples are gemcitabine, carboplatin, and cyclophosphamide.

\section{Platform comparison}

Although putative neoantigens may be accurately predicted, selected, and validated, directing those epitopes to the proper immune cells to elicit tumor-specific CD4+ and CD8+ responses is also essential. ${ }^{53}$ DNA-, RNA-, synthetic long peptide-, and dendritic cell-based vaccines constitute the delivery platforms used in current clinical trials. Table 2 allows comparison of the underlying platform mechanisms, formulations, advantages, and disadvantages. Amongst the four, peptide-based vaccines are found in the plurality of clinical trials. Here, polyinosinicpolycytidylic acid and poly-L-lysine (poly-ICLC), is the most widely-used adjuvant for peptide vaccines. Such treatment mimics dsRNA, a product of various viral infections, to activate TLR3. ${ }^{54}$ The immune response driven by peptide vaccines is currently unpredictable at best, and often weak or temporary, ${ }^{55}$ and thus requires the addition of adjuvants. DNA and mRNA platforms are believed to be inherently more immunogenic as these molecules naturally provoke the innate immune system, ${ }^{56,57}$ however, incorporation of modified nucleoside into these molecules, such as pseudouridine for mRNA, can help balance immune activation versus antigen stability and expression. ${ }^{58}$ DNA vaccines cloned into a plasmid are designed for host nuclear retention without integration into the host genome, while mRNA is directed to the cytoplasm for translation. ${ }^{59}$ However, mRNA can easily be degraded by extracellular RNases. As a result, protective strategies, such as liposome- and nanoparticle-encasement, are essential for efficient RNA delivery. ${ }^{57}$ Additionally, lipid nanoparticle encased pseudouridylated mRNA has been shown to have an adjuvant effect, stimulating both $\mathrm{T}$ helper and germinal $\mathrm{B}$ cells. ${ }^{58}$ Given these potentially strong immune responses as well as a low risk of genomic integration, mRNA-based vaccines are becoming increasingly popular, with a number of prominent biotech companies favoring this approach. In collaboration with BioNTech, Sahin's group has been assessing safety and efficacy of TAA-based and TSA-based mRNA vaccines in more than 30 TNBC patients (NCT02316457). Moderna, a biotech whose rapid development of a potential Covid-19 vaccine has made headlines, has led a clinical trial introducing mRNA vaccines with and without pembrolizumab to 33 resected solid

Table 2. The platforms by which neoantigens may be delivered.

\begin{tabular}{|c|c|c|c|c|c|}
\hline Platform & Mechanisms & Formulations & Pros & Cons & $\begin{array}{l}\text { Clinical Trial } \\
\text { Example }\end{array}$ \\
\hline DNA & $\begin{array}{l}\text { - STING/TBK1/IRF3 } \\
\text { - TLR } 9\end{array}$ & Cloned plasmid & $\begin{array}{l}\text { - Amplification to multiple } \\
\text { peptides } \\
\text { - Polyepitopes in a plasmid } \\
\text { available }\end{array}$ & $\begin{array}{l}\text { - Possibly integrates into } \\
\text { genome } \\
\text { - Production involves bacteria }\end{array}$ & $\begin{array}{l}\text { - NCT03122106 } \\
\text { (phase I ongoing) } \\
\text { - NCT03199040 } \\
\text { (phase I ongoing) }\end{array}$ \\
\hline mRNA & - $\operatorname{TLR} 3,7,8$ & $\begin{array}{l}\text { - Naked } \\
\text { - Liposomes } \\
\text { - Nanoparticles } \\
\text { - Viruses }\end{array}$ & $\begin{array}{l}\text { - Do not integrate into genome } \\
\text { - Polyepitopes in an mRNA } \\
\text { available } \\
\text { - mRNA may have } \\
\text { "self-adjuvant" effects }\end{array}$ & $\begin{array}{l}\text { - Instability: protection from } \\
\text { RNases required } \\
\text { - In vitro transcription: } \\
\text { expensive }\end{array}$ & $\begin{array}{l}\text { - NCT03908671 } \\
\text { (phase I ongoing) } \\
\text { - NCT03480152 } \\
\text { (phase I: no "serious } \\
\text { adverse events"; } \\
\text { phase II: ongoing) }\end{array}$ \\
\hline Peptide & $\begin{array}{l}\text { - Internalization by } \\
\text { DCs } \\
\text { - Cross-presentation } \\
\text { under appropriate } \\
\text { activation and } \\
\text { processing }\end{array}$ & $\begin{array}{l}\text { Synthetic long } \\
\text { peptide with } \\
\text { poly-ICLC }\end{array}$ & $\begin{array}{l}\text { - Stable } \\
\text { - Safe } \\
\text { - More data from clinical trials }\end{array}$ & $\begin{array}{l}\text { - Low immunogenicity } \\
\text { - Multiple epitope production } \\
\text { required } \\
\text { - } \text { Adjuvant required }\end{array}$ & 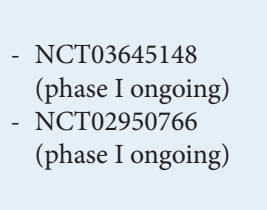 \\
\hline DC & $\begin{array}{l}\text { - Antigen presentation } \\
\text { to } \mathrm{T} \text { cells }\end{array}$ & $\begin{array}{l}\text { Mature and } \\
\text { activated DCs }\end{array}$ & $\begin{array}{l}\text { - Possibly the best way to } \\
\text { prime CD8 and CD4 T cells }\end{array}$ & $\begin{array}{l}\text { - Expensive } \\
\text { - Time-consuming } \\
\text { - In vitro peptide loading } \\
\text { required }\end{array}$ & $\begin{array}{l}\text { - NCT04105582 } \\
\text { (phase I ongoing) } \\
\text { - NCT04078269 } \\
\text { (phase I ongoing) }\end{array}$ \\
\hline
\end{tabular}


tumor patients (NCT03313778). Clinical outcomes and T-cell responses were sufficient to proceed to a phase-2 trial on high-risk melanoma patients (NCT03897881). Intuitively, DC-primed vaccines might offer the optimal strategy to initiate $\mathrm{T}$ cell responses, bypassing steps from DNA transcription to peptide presentation. Not surprisingly though, cost and time constraints may significantly limit the benefits of such an approach. ${ }^{55}$ Hopefully, the current generation of clinical trials will generate clarity as to the optimal platforms for particular cancers.

\section{Limitations of neoantigen-based therapy}

We'd be remiss in failing to mention possible stumbling blocks. As seen above, identification of neoantigens remains a fluid area of research, with little consensus as to the neoantigen sources that are most immunogenic, while neoantigen combination therapies and delivery methods are far from optimized. Additionally, regulatory hurdles are substantial; current practices are centered on drugs with specific molecular formulas that have undergone years of testing. Such a paradigm obviously cannot be applied to neoantigen development, where personalized treatments must be generated in a matter of weeks. ${ }^{60}$

At the biological level, a number of studies suggest that many immunogenic neoantigen candidates are passengers, not drivers of cancer development. ${ }^{61}$ Additionally, driver mutations may be selected for weak immunogenicity. ${ }^{62}$ The heterogeneity of cancer tissue means that even strongly immunogenic neoantigens may fail to elicit whole tumor destruction. Also, loss of HLA heterozygosity (HLA-LOH) is a common immune-evasion tactic in cancer. In one study, loss of HLA was seen in $40 \%$ of non-small-lung cell cancer samples, ${ }^{63}$ eliminating some degree of neoantigen presentation capacity. Not surprisingly, however, organisms do not necessarily take kindly to HLA-LOH, which may stimulate the activity of NK cells. ${ }^{64}$ More often, it has been argued, HLA is overexpressed in cancer. ${ }^{65}$

\section{Conclusions}

We've highlighted sources of neoantigens and the status of clinical trials as particularly fast-moving subjects. A number of other topics deserve at least a mention. First, a glimpse at these clinical trials shows that neoantigen-therapies are predominantly administered in combination with immunomodulatory drugs. While PD-1/PD-L1 inhibitors are currently dominant, numerous other immuno-inhibitory molecules (e.g. TIM-3, VISTA) and stimulatory molecules (e.g. OX40, CD40) are currently being targeted by next-generation drugs. ${ }^{66}$ Thus optimization of therapy combinations remains a fluid area of research. Secondly, while it is generally thought that neoantigen immunogenicity correlates with HLA-binding affinity, ${ }^{67}$ there are some indications that poorly-binding neoantigens may still elicit immune responses. ${ }^{68,69}$ Delineation of the precise neoantigen characteristics that elicit immunogenicity will likely reveal nuance. Finally, we should point out that there is no reason that cancer-unique antigens must be confined to peptides. Some HLA variants (e.g. HLA-E, MR1) are capable of presenting lipids and metabolites, some of which may be unique to cancers and/or infections. ${ }^{70}$
Practically speaking, the expenses involved in pipelines that require multiple "omics" protocols, the rapid output of peptides or peptide-coding RNA/DNA vectors, and treatment with complementary immunotherapeutics, cannot be denied. It is comforting to note, however, that labs around the world are working diligently to minimize such costs, emphasizing compassion over profit.

\section{Competing interests}

The authors declare no conflicts of interest.

\section{Acknowledgments}

$\mathrm{KH}$ is supported by Rachadaphiseksompot Fund for Postdoctoral Fellowship, Chulalongkorn University, Thailand. This work was supported by Chulalongkorn Academic Advancement into Its $2^{\text {nd }}$ Century (CUAASC) Project. The authors wish to thank Tossapon Wongtangprasert for assistance with graphics.

\section{References}

1. Morgan RA, Chinnasamy N, Abate-Daga D, Gros A, Robbins PF, Zheng Z, et al. Cancer regression and neurological toxicity following anti-MAGE-A3 TCR gene therapy. J Immunother. 2013;36(2):133-51.

2. Morgan RA, Yang JC, Kitano M, Dudley ME, Laurencot CM, Rosenberg SA. Case report of a serious adverse event following the administration of T cells transduced with a chimeric antigen receptor recognizing ERBB2. Mol Ther. 2010;18(4):843-51.

3. Brentjens R, Yeh R, Bernal Y, Riviere I, Sadelain M. Treatment of chronic lymphocytic leukemia with genetically targeted autologous $\mathrm{T}$ cells: case report of an unforeseen adverse event in a phase I clinical trial. Mol Ther. 2010;18(4):666-8.

4. Hsieh CS, Lee HM, Lio CW. Selection of regulatory T cells in the thymus. Nat Rev Immunol. 2012;12(3):157-67.

5. Xing Y, Hogquist KA. T-cell tolerance: central and peripheral. Cold Spring Harb Perspect Biol. 2012;4(6).

6. Robbins PF, Lu YC, El-Gamil M, Li YF, Gross C, Gartner J, et al. Mining exomic sequencing data to identify mutated antigens recognized by adoptively transferred tumor-reactive T cells. Nat Med. 2013;19(6):747-52.

7. McGranahan N, Furness AJ, Rosenthal R, Ramskov S, Lyngaa R, Saini SK, et al. Clonal neoantigens elicit $\mathrm{T}$ cell immunoreactivity and sensitivity to immune checkpoint blockade. Science. 2016;351(6280):1463-9.

8. Snyder A, Makarov V, Merghoub T, Yuan J, Zaretsky JM, Desrichard A, et al. Genetic basis for clinical response to CTLA-4 blockade in melanoma. N Engl J Med. 2014;371(23):2189-99.

9. Gerlinger M, Rowan AJ, Horswell S, Math M, Larkin J, Endesfelder D, et al. Intratumor heterogeneity and branched evolution revealed by multiregion sequencing. N Engl J Med. 2012;366(10):883-92.

10. Slaney CY, Kershaw MH, Darcy PK. Trafficking of T cells into tumors. Cancer Res. 2014;74(24):7168-74.

11. Farhood B, Najafi M, Mortezaee K. CD8(+) cytotoxic T lymphocytes in cancer immunotherapy: A review. J Cell Physiol. 2019;234(6):8509-21.

12. Rajasagi M, Shukla SA, Fritsch EF, Keskin DB, DeLuca D, Carmona E, et al. Systematic identification of personal tumor-specific neoantigens in chronic lymphocytic leukemia. Blood. 2014;124(3):453-62.

13. Cibulskis K, Lawrence MS, Carter SL, Sivachenko A, Jaffe D, Sougnez $\mathrm{C}$, et al. Sensitive detection of somatic point mutations in impure and heterogeneous cancer samples. Nat Biotechnol. 2013;31(3):213-9.

14. Kiyotani K, Mai TH, Nakamura Y. Comparison of exome-based HLA class I genotyping tools: identification of platform-specific genotyping errors. J Hum Genet. 2017;62(3):397-405.

15. Hao Y, Yan M, Heath BR, Lei YL, Xie Y. Fast and robust deconvolution of tumor infiltrating lymphocyte from expression profiles using least trimmed squares. PLoS Comput Biol. 2019;15(5):e1006976.

16. Giannakis M, Mu XJ, Shukla SA, Qian ZR, Cohen O, Nishihara R, et al Genomic Correlates of Immune-Cell Infiltrates in Colorectal Carcinoma. Cell Rep. 2016;15(4):857-65. 
17. Turajlic S, Litchfield $\mathrm{K}, \mathrm{Xu} \mathrm{H}$, Rosenthal $\mathrm{R}$, McGranahan $\mathrm{N}$, Reading $\mathrm{JL}$, et al. Insertion-and-deletion-derived tumour-specific neoantigens and the immunogenic phenotype: a pan-cancer analysis. Lancet Oncol. 2017;18(8):1009-21.

18. Koster J, Plasterk RHA. A library of Neo Open Reading Frame peptides (NOPs) as a sustainable resource of common neoantigens in up to $50 \%$ of cancer patients. Sci Rep. 2019;9(1):6577.

19. Bjerregaard AM, Nielsen M, Jurtz V, Barra CM, Hadrup SR, Szallasi Z, et al. An Analysis of Natural T Cell Responses to Predicted Tumor Neoepitopes. Front Immunol. 2017;8:1566.

20. Wei Z, Zhou C, Zhang Z, Guan M, Zhang C, Liu Z, et al. The Landscape of Tumor Fusion Neoantigens: A Pan-Cancer Analysis. iScience. 2019;21: 249-60

21. Yang W, Lee KW, Srivastava RM, Kuo F, Krishna C, Chowell D, et al. Immunogenic neoantigens derived from gene fusions stimulate $\mathrm{T}$ cell responses. Nat Med. 2019;25(5):767-75.

22. Makita M, Azuma T, Hamaguchi H, Niiya H, Kojima K, Fujita S, et al. Leukemia-associated fusion proteins, dek-can and bcr-abl, represent immunogenic HLA-DR-restricted epitopes recognized by fusion peptide -specific CD4+ T lymphocytes. Leukemia. 2002;16(12):2400-7.

23. Jayasinghe RG, Cao S, Gao Q, Wendl MC, Vo NS, Reynolds SM, et al. Systematic Analysis of Splice-Site-Creating Mutations in Cancer. Cell Rep. 2018;23(1):270-81 e3.

24. Kahles A, Lehmann KV, Toussaint NC, Huser M, Stark SG, Sachsenberg T, et al. Comprehensive Analysis of Alternative Splicing Across Tumors from 8,705 Patients. Cancer Cell. 2018;34(2):211-24 e6.

25. Smart AC, Margolis CA, Pimentel H, He MX, Miao D, Adeegbe D, et al. Intron retention is a source of neoepitopes in cancer. Nat Biotechnol. 2018;36(11):1056-8.

26. Zhao B, Pritchard JR. Evolution of the nonsense-mediated decay pathway is associated with decreased cytolytic immune infiltration. PLoS Comput Biol. 2019;15(10):e1007467.

27. Laumont CM, Vincent K, Hesnard L, Audemard E, Bonneil E, Laverdure JP, et al. Noncoding regions are the main source of targetable tumor-specific antigens. Sci Transl Med. 2018;10(470).

28. Chong C, Muller M, Pak H, Harnett D, Huber F, Grun D, et al. Integrated proteogenomic deep sequencing and analytics accurately identify non -canonical peptides in tumor immunopeptidomes. Nat Commun. 2020; 11(1):1293.

29. Smith CC, Beckermann KE, Bortone DS, De Cubas AA, Bixby LM, Lee SJ, et al. Endogenous retroviral signatures predict immunotherapy response in clear cell renal cell carcinoma. J Clin Invest. 2018;128(11):4804-20.

30. Christofi T, Zaravinos A. RNA editing in the forefront of epitranscriptomics and human health. J Transl Med. 2019;17(1):319.

31. Peng X, Xu X, Wang Y, Hawke DH, Yu S, Han L, et al. A-to-I RNA Editing Contributes to Proteomic Diversity in Cancer. Cancer Cell. 2018; 33(5):817-28 e7

32. Abelin JG, Trantham PD, Penny SA, Patterson AM, Ward ST, Hildebrand $\mathrm{WH}$, et al. Complementary IMAC enrichment methods for HLA-associated phosphopeptide identification by mass spectrometry. Nat Protoc. 2015; 10(9):1308-18.

33. Solleder M, Guillaume P, Racle J, Michaux J, Pak HS, Muller M, et al. Mass Spectrometry Based Immunopeptidomics Leads to Robust Predictions of Phosphorylated HLA Class I Ligands. Mol Cell Proteomics. 2020;19(2): 390-404.

34. Malaker SA, Penny SA, Steadman LG, Myers PT, Loke JC, Raghavan M, et al. Identification of Glycopeptides as Posttranslationally Modified Neoantigens in Leukemia. Cancer Immunol Res. 2017;5(5):376-84.

35. Mylonas R, Beer I, Iseli C, Chong C, Pak HS, Gfeller D, et al. Estimating the Contribution of Proteasomal Spliced Peptides to the HLA-I Ligandome. Mol Cell Proteomics. 2018;17(12):2347-57.

36. Rolfs Z, Solntsev SK, Shortreed MR, Frey BL, Smith LM. Global Identification of Post-Translationally Spliced Peptides with Neo-Fusion. J Proteome Res. 2019;18(1):349-58.

37. Liepe J, Marino F, Sidney J, Jeko A, Bunting DE, Sette A, et al. A large fraction of HLA class I ligands are proteasome-generated spliced peptides. Science. 2016;354(6310):354-8.

38. Liepe J, Sidney J, Lorenz FKM, Sette A, Mishto M. Mapping the MHC Class I-Spliced Immunopeptidome of Cancer Cells. Cancer Immunol Res. 2019;7(1):62-76.

39. Grimaldi A, Cammarata I, Martire C, Focaccetti C, Piconese S, Buccilli $\mathrm{M}$, et al. Combination of chemotherapy and PD-1 blockade induces T cell responses to tumor non-mutated neoantigens. Commun Biol. 2020;3(1): 85 .
40. Schietinger A, Philip M, Liu RB, Schreiber K, Schreiber H. Bystander killing of cancer requires the cooperation of CD4(+) and CD8(+) T cells during the effector phase. J Exp Med. 2010;207(11):2469-77.

41. Yadav M, Jhunjhunwala S, Phung QT, Lupardus P, Tanguay J, Bumbaca $\mathrm{S}$, et al. Predicting immunogenic tumour mutations by combining mass spectrometry and exome sequencing. Nature. 2014;515(7528):572-6.

42. Castle JC, Kreiter S, Diekmann J, Lower M, van de Roemer N, de Graaf J, et al. Exploiting the mutanome for tumor vaccination. Cancer Res. 2012;72(5):1081-91.

43. Kreiter S, Vormehr M, van de Roemer N, Diken M, Lower M, Diekmann J, et al. Mutant MHC class II epitopes drive therapeutic immune responses to cancer. Nature. 2015;520(7549):692-6.

44. Bayat Mokhtari R, Homayouni TS, Baluch N, Morgatskaya E, Kumar $\mathrm{S}$, Das B, et al. Combination therapy in combating cancer. Oncotarget. 2017;8(23):38022-43.

45. Zhao J, Chen Y, Ding ZY, Liu JY. Safety and Efficacy of Therapeutic Cancer Vaccines Alone or in Combination With Immune Checkpoint Inhibitors in Cancer Treatment. Front Pharmacol. 2019;10:1184.

46. Azoury SC, Straughan DM, Shukla V. Immune Checkpoint Inhibitors for Cancer Therapy: Clinical Efficacy and Safety. Curr Cancer Drug Targets. 2015;15(6):452-62.

47. D’Alise AM, Leoni G, Cotugno G, Troise F, Langone F, Fichera I, et al Adenoviral vaccine targeting multiple neoantigens as strategy to eradicate large tumors combined with checkpoint blockade. Nat Commun. 2019; 10(1):2688

48. Massarelli E, William W, Johnson F, Kies M, Ferrarotto R, Guo M, et al. Combining Immune Checkpoint Blockade and Tumor-Specific Vaccine for Patients With Incurable Human Papillomavirus 16-Related Cancer: A Phase 2 Clinical Trial. JAMA Oncol. 2019;5(1):67-73.

49. Kuai R, Ochyl LJ, Bahjat KS, Schwendeman A, Moon JJ. Designer vaccine nanodiscs for personalized cancer immunotherapy. Nat Mater. 2017; 16(4):489-96.

50. Rizvi NA, Hellmann MD, Snyder A, Kvistborg P, Makarov V, Havel JJ, et al. Cancer immunology. Mutational landscape determines sensitivity to PD-1 blockade in non-small cell lung cancer. Science. 2015;348(6230):124-8.

51. Subudhi SK, Vence L, Zhao H, Blando J, Yadav SS, Xiong Q, et al Neoantigen responses, immune correlates, and favorable outcomes after ipilimumab treatment of patients with prostate cancer. Sci Transl Med. 2020;12(537).

52. Emens LA, Middleton G. The interplay of immunotherapy and chemotherapy: harnessing potential synergies. Cancer Immunol Res. 2015; 3(5):436-43

53. Guo Y, Lei K, Tang L. Neoantigen Vaccine Delivery for Personalized Anticancer Immunotherapy. Front Immunol. 2018;9:1499.

54. Liu H, Zha Y, Choudhury N, Malnassy G, Fulton N, Green M, et al. WT1 peptide vaccine in Montanide in contrast to poly ICLC, is able to induce WT1-specific immune response with TCR clonal enrichment in myeloid leukemia. Exp Hematol Oncol. 2018;7:1

55. Li L, Goedegebuure SP, Gillanders WE. Preclinical and clinical development of neoantigen vaccines. Ann Oncol. 2017;28(suppl_12): xii11-xii7.

56. Coban C, Koyama S, Takeshita F, Akira S, Ishii KJ. Molecular and cellular mechanisms of DNA vaccines. Hum Vaccin. 2008;4(6):453-6.

57. Pardi N, Hogan MJ, Porter FW, Weissman D. mRNA vaccines - a new era in vaccinology. Nat Rev Drug Discov. 2018;17(4):261-79.

58. Zhang C, Maruggi G, Shan H, Li J. Advances in mRNA Vaccines for Infectious Diseases. Front Immunol. 2019;10:594

59. Liu MA. A Comparison of Plasmid DNA and mRNA as Vaccine Technologies. Vaccines (Basel). 2019;7(2).

60. Hacohen N, Fritsch EF, Carter TA, Lander ES, Wu CJ. Getting personal with neoantigen-based therapeutic cancer vaccines. Cancer Immunol Res. 2013;1(1):11-5.

61. Charoentong P, Finotello F, Angelova M, Mayer C, Efremova M, Rieder D, et al. Pan-cancer Immunogenomic Analyses Reveal Genotype -Immunophenotype Relationships and Predictors of Response to Checkpoint Blockade. Cell Rep. 2017;18(1):248-62.

62. Nejo T, Matsushita H, Karasaki T, Nomura M, Saito K, Tanaka S, et al. Reduced Neoantigen Expression Revealed by Longitudinal Multiomics as a Possible Immune Evasion Mechanism in Glioma. Cancer Immunol Res. 2019;7(7):1148-61.

63. McGranahan N, Rosenthal R, Hiley CT, Rowan AJ, Watkins TBK, Wilson GA, et al. Allele-Specific HLA Loss and Immune Escape in Lung Cancer Evolution. Cell. 2017;171(6):1259-71 e11. 
64. Boudreau JE, Hsu KC. Natural killer cell education in human health and disease. Curr Opin Immunol. 2018;50:102-11.

65. Freudenmann LK, Marcu A, Stevanovic S. Mapping the tumour human leukocyte antigen (HLA) ligandome by mass spectrometry. Immunology. 2018;154(3):331-45.

66. Dempke WCM, Fenchel K, Uciechowski P, Dale SP. Second- and third -generation drugs for immuno-oncology treatment-The more the better? Eur J Cancer. 2017;74:55-72.

67. Engels B, Engelhard VH, Sidney J, Sette A, Binder DC, Liu RB, et al. Relapse or eradication of cancer is predicted by peptide-major histocompatibility complex affinity. Cancer Cell. 2013;23(4):516-26.
68. Duan F, Duitama J, Al Seesi S, Ayres CM, Corcelli SA, Pawashe AP, et al. Genomic and bioinformatic profiling of mutational neoepitopes reveals new rules to predict anticancer immunogenicity. J Exp Med. 2014;211(11): 2231-48.

69. Ebrahimi-Nik H, Michaux J, Corwin WL, Keller GL, Shcheglova T, Pak $\mathrm{H}$, et al. Mass spectrometry driven exploration reveals nuances of neoepitope-driven tumor rejection. JCI Insight. 2019;5.

70. Ogg G, Cerundolo V, McMichael AJ. Capturing the antigen landscape: HLA-E, CD1 and MR1. Curr Opin Immunol. 2019;59:121-9. 